\title{
Single quantum dot emission at telecom wavelengths from metamorphic InAs/InGaAs nanostructures grown on GaAs substrates
}

\author{
L. Seravalli, ${ }^{1, a)}$ G. Trevisi, ${ }^{1}$ P. Frigeri, ${ }^{1}$ D. Rivas, ${ }^{2}$ G. Muñoz-Matutano, ${ }^{2}$ I. Suárez, ${ }^{2}$ B. Alén, ${ }^{2}$ \\ J. Canet-Ferrer, ${ }^{2}$ and J. P. Martínez-Pastor ${ }^{2}$ \\ ${ }^{1}$ CNR-IMEM Institute, Parco delle Scienze 37a, I-43100 Parma, Italy \\ ${ }^{2}$ UMDO (Unidad Asociada al IMM-CSIC), Instituto de Ciencia de los Materiales, Universidad de Valencia, \\ P.O. Box 22085, 46071 Valencia, Spain
}

(Received 21 February 2011; accepted 9 April 2011; published online 29 April 2011; corrected 12 May 2011)

\begin{abstract}
We report on the growth by molecular beam epitaxy and the study by atomic force microscopy and photoluminescence of low density metamorphic InAs/InGaAs quantum dots. subcritical InAs coverages allow to obtain $10^{8} \mathrm{~cm}^{-2}$ dot density and metamorphic $\operatorname{In}_{\mathrm{x}} \mathrm{Ga}_{1-\mathrm{x}} \mathrm{As}(\mathrm{x}=0.15,0.30)$ confining layers result in emission wavelengths at $1.3 \mu \mathrm{m}$. We discuss optimal growth parameters and demonstrate single quantum dot emission up to $1350 \mathrm{~nm}$ at low temperatures, by distinguishing the main exciton complexes in these nanostructures. Reported results indicate that metamorphic quantum dots could be valuable candidates as single photon sources for long wavelength telecom windows. () 2011 American Institute of Physics. [doi:10.1063/1.3584132]
\end{abstract}

Self-assembled InAs/GaAs semiconductor quantum dots (QDs) are investigated as viable sources of single photons for quantum cryptography and quantum communication, ${ }^{1,2}$ thanks to the wide degree of tunability of emission and the growth on GaAs substrates. ${ }^{3}$ However, a surface density of a few dots per micro square meter is necessary to allow detection of single photons, requiring ad hoc growth procedures. $^{4,5}$ In molecular beam epitaxy (MBE), the deposition of subcritical InAs coverages followed by postgrowth annealing (PGA) is an useful approach, ${ }^{6,7}$ but to match the QD emission with the $1.3 \mu \mathrm{m}$ telecom window, it is necessary to engineer the InAs/GaAs structure, for example, adding InGaAs or GaSb capping layers. ${ }^{4,5}$, To extend the emission up to $1.55 \mu \mathrm{m}$, the growth of QDs on metamorphic buffers has been useful for high density structures. ${ }^{9-11}$ This approach has been also considered for low density QDs; the substrate rotation is stopped during InAs deposition but single QD emission is observed only in a restricted area. ${ }^{12}$

Here we report on an original approach to obtain single QD emission from metamorphic structures, relying on subcritical InAs coverages deposited on relaxed InGaAs layers, that allows to obtain $10^{8} \mathrm{~cm}^{-2}$ QD density, homogenously distributed on the surface. As discussed in some works, ${ }^{10,12}$ growing QDs on metamorphic InGaAs is different from growing them on GaAs, due to the reduced mismatch between QDs and lower confining layer (LCL) and the dissimilar surface. Thus, the aim of the letter is the study of single QD emission and of peculiarities of this system in comparison with the InAs/GaAs one.

MBE-grown structures consist of (i) $100 \mathrm{~nm} \mathrm{GaAs}$ buffer, (ii) $500 \mathrm{~nm} \operatorname{In}_{\mathrm{x}} \mathrm{Ga}_{1-\mathrm{x}}$ As LCL deposited at $400{ }^{\circ} \mathrm{C}$, (iii) $5 \mathrm{~nm}$ of GaAs to smooth the surface, (iv) $\theta$ monolayer (ML) InAs layer at $490{ }^{\circ} \mathrm{C}$ followed by a PGA time $\tau$ under As flux, and (v) $20 \mathrm{~nm} \mathrm{In}_{\mathrm{x}} \mathrm{Ga}_{1-\mathrm{x}}$ As layer grown at $360{ }^{\circ} \mathrm{C}$. LCL compositions of $\mathrm{x}=0.15$ and $\mathrm{x}=0.30$ result in a QDLCL mismatch of $6.34 \%$ and $5.19 \%$, respectively, as calculated on the basis of the Mareè theory, ${ }^{13}$ and experimentally

${ }^{a)}$ Electronic mail: seravall@imem.cnr.it. confirmed by different techniques in Refs. 14 and 15. Reflection high energy electron diffraction patterns were used to determine the two-dimensional to three-dimensional (3D) InAs critical thickness $\left(\theta_{\mathrm{c}}\right)$ on InGaAs LCL and to monitor the evolution of subcritical layers during the PGA. Atomic force microscopy (AFM) measurements were performed on uncapped QD structures. The microphotoluminescence $(\mu \mathrm{PL})$ was measured at $4 \mathrm{~K}$ with 800 and $830 \mathrm{~nm}$ exciting light from a Ti:sapphire mode-locked laser, using a confocal microscope to focus the laser spot. The $\mu \mathrm{PL}$ signal was collected with a single-mode fiber and coupled into a monochromator, where light was dispersed into a cooled InGaAs photodiode array device with $90 \%$ quantum efficiency.

The value of $\theta_{\mathrm{c}}$ has been measured to be $1.8 \mathrm{ML}$ for $\mathrm{x}=0.15$ and $2.2 \mathrm{ML}$ for $\mathrm{x}=0.30$. Then InAs coverages below these values were used, to derive optimal values of subcritical coverage $\theta_{\text {sub }}$ and $\tau$ resulting in low QD densities, as

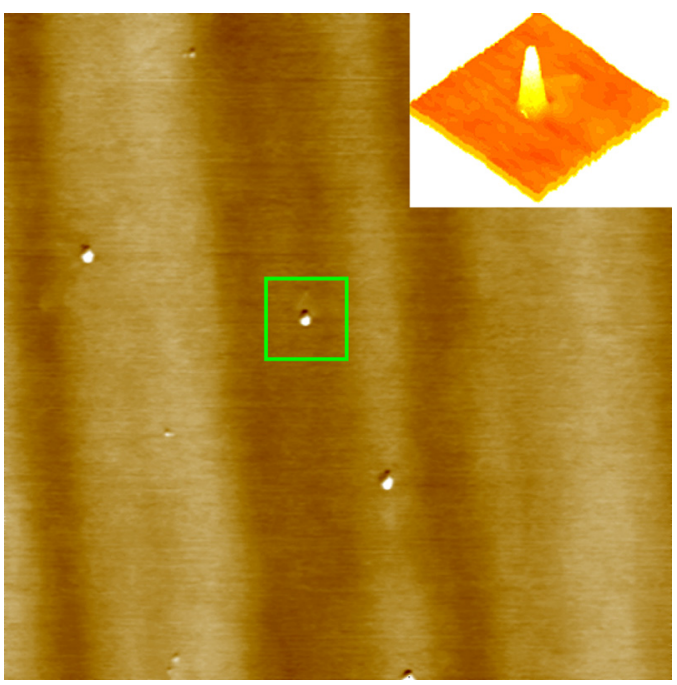

FIG. 1. (Color online) AFM image of $x=0.15$ sample. Square $(250$ $\times 250 \mathrm{~nm}^{2}$ ) highlights a single QD of height $9.8 \mathrm{~nm}$, whose 3D image is shown in the inset. 

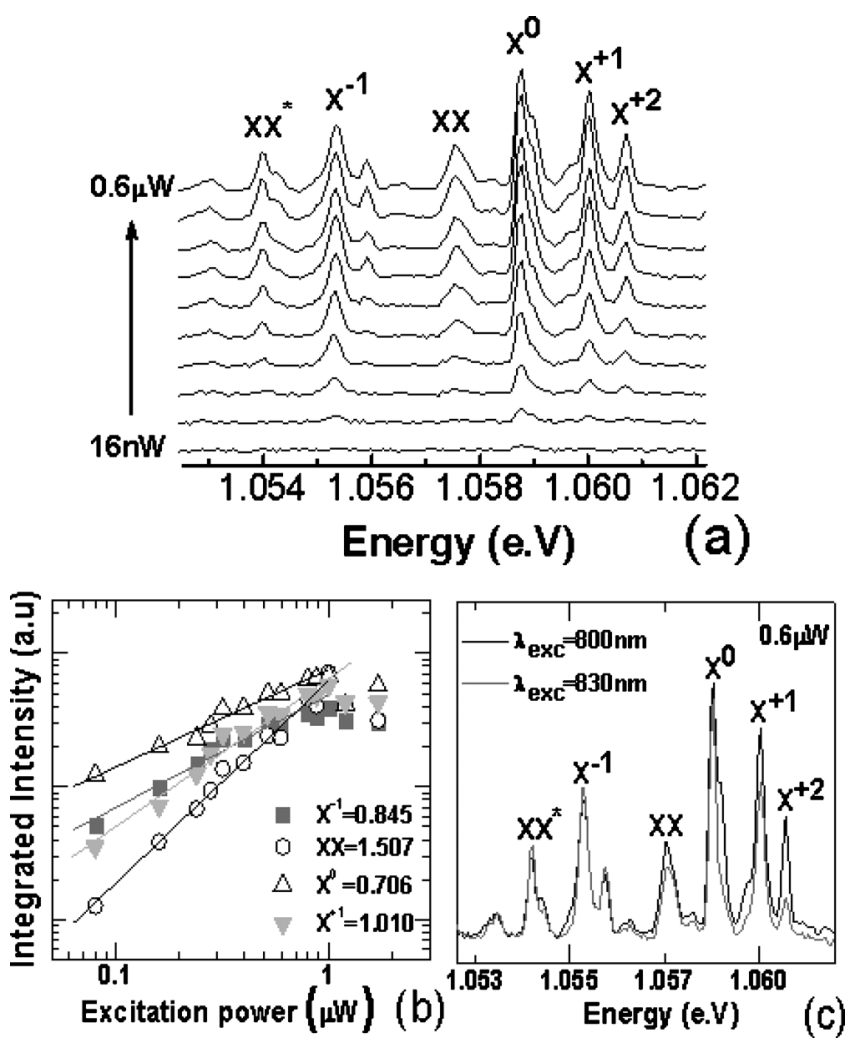

FIG. 2. $\mu \mathrm{PL}$ spectra form a single $\mathrm{QD}$ emitting at around $1171 \mathrm{~nm}$ in a sample with a LCL of low indium content $(x=0.15)$ measured at $4 \mathrm{~K}$. (a) Power evolution of the $\mu \mathrm{PL}$ (from 16 to $600 \mathrm{nW}$ ), (b) Integrated intensity with respect to the excitation power, (c) Spectra using selective optical excitation (800 and $830 \mathrm{~nm}$ ).

shown in Fig. 1 for $\mathrm{x}=0.15$ sample, where the typical surface roughening of relaxed InGaAs surfaces is also noticeable. Structure with $\mathrm{x}=0.30$ have very similar morphological properties, as shown and discussed in Ref. 16. Relevant values for $\mathrm{x}=0.15$ were $\theta_{\text {sub }}=1.5 \mathrm{ML}$ and $\tau=22 \mathrm{~s}$ while for $\mathrm{x}=0.30$, values were $\theta_{\text {sub }}=1.8 \mathrm{ML}$ and $\tau=300 \mathrm{~s}$. It can be noted that the optimal value for the difference between $\theta_{\text {sub }}$ and $\theta_{\mathrm{c}}$ depends on $\mathrm{x}(0.3 \mathrm{ML}$ for $\mathrm{x}=0.15$ and $0.4 \mathrm{ML}$ for $\mathrm{x}=0.30$ ) and is larger than the value usually reported for the InAs/GaAs system of 0.1-0.15 ML. ${ }^{6,17}$ These experimental results could be explained by modified kinetics of adatoms due to different composition, strain fields, and morphology of the surface, strengthening the notion that InAs QD deposition on InGaAs surfaces is intrinsically different from growth on GaAs ones. ${ }^{10,18-20}$

Figures 2 and 3 show $\mu \mathrm{PL}$ from samples with $\mathrm{x}=0.15$ and $x=0.30$ LCLs, respectively, under different power excitations. At low excitation power both spectra show localized narrow lines characteristic of single QD emission. In structures with $\mathrm{x}=0.30 \mathrm{LCL}$ the emission is redshifted, due to reduction in QD strain and of QD-CL band discontinuities. ${ }^{21}$ Thus, metamorphic nanostructures are able to emit one single photon into the second telecommunication window band $(1.3 \mu \mathrm{m})$ and the emission wavelength may be tuned by changing the composition of the LCL layers.

Figure 2(a) shows the power excitation dependence $\mu \mathrm{PL}$ from the $\mathrm{X}=0.15$ sample. Typical excitonic $\left(\mathrm{X}^{\mathrm{n}}\right)$ and biexcitonic $\left(\mathrm{XX}^{\mathrm{n}}\right)$ lines can be clearly identified via the integrated intensity power dependence [Fig. 2(b)], ${ }^{22}$ the coulombic bonding energy and the selective optical pumping effect [Fig.
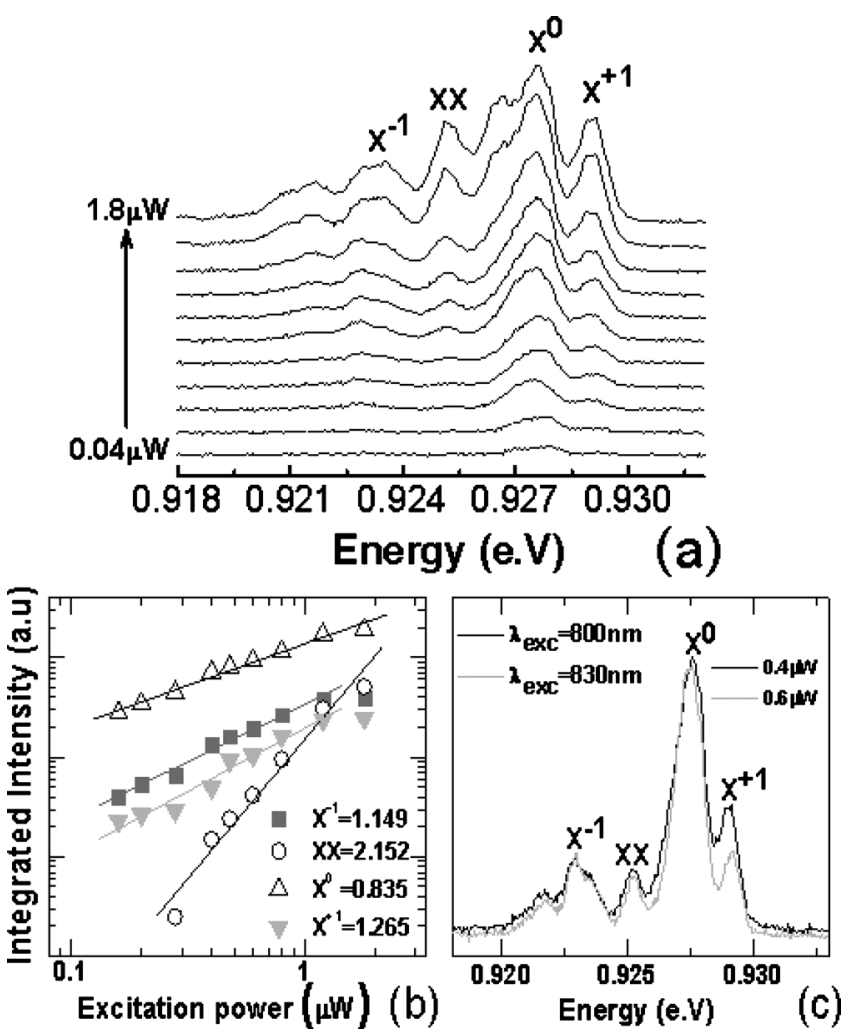

FIG. 3. $\mu \mathrm{PL}$ spectra form a single $\mathrm{QD}$ emitting at around $1336 \mathrm{~nm}$ in a sample with a LCL of high indium content $(x=0.30)$ measured at $4 \mathrm{~K}$. (a) Power evolution of the $\mu \mathrm{PL}$ (from $40 \mathrm{nW}$ to $1.8 \mu \mathrm{W}$ ), (b) Integrated intensity with respect to the excitation power, (c) Spectra using selective optical excitation $(800$ and $830 \mathrm{~nm}$ ).

2(c)] associated to local unintentional impurities. ${ }^{23-25} \mathrm{Neu}-$ tral exciton $\left(\mathrm{X}^{0}\right)$ is identified at $1.0587 \mathrm{eV}$ (around $1170 \mathrm{~nm}$ ), where the smaller than unity $(0.705)$ slope of the transition comes from the statistical measurement process and the existence of the residual impurities. ${ }^{26}$ The line centered at $1.0575\left(1.2 \mathrm{meV}\right.$ redshifted from $\left.\mathrm{X}^{0}\right)$ was assigned to neutral biexciton $(\mathrm{XX})$, as its slope (1.455) is double of neutral exciton. Positive and negative trions $\left(\mathrm{X}^{+1}\right.$ at $1.0600 \mathrm{eV}$ and $\mathrm{X}^{-1}$ at $1.0553 \mathrm{eV}$ ) are identified by a slope close to one and the greater importance of the negative trion lines, when the excitation energy is resonant with residual acceptor impurity levels (see Ref. 23 for more information on charge initialization by selective optical pumping). In InAs/GaAs QDs emitting in the same spectral region positive and negative trions are usually blue and redshifted with respect to $\mathrm{X}^{0}$ by 1-2 meV and 5-7 meV, respectively. ${ }^{23,27}$ Thus, results obtained here are in agreement with the ones measured in previous works. Finally, weak lines observed at 1.0560 and $1.0540 \mathrm{eV}$ for medium-high excitation powers can have their origin on charged biexcitons and hence are labeled as $\mathrm{XX}^{*}$ but there is not enough information for a clearer identification. In this sample $(x=0.15)$ we have found examples of many other single QDs, emitting from 1080 to $1210 \mathrm{~nm}$.

In the sample with $\mathrm{x}=0.30 \mathrm{LCL} \mathrm{QD}$ emission is found in the range 1200-1350 $\mathrm{nm}$ and excitonic complexes (Fig. 3) can be identified using the same methods described above, based on the power dependence [Figs. 3(a) and 3(b)]. In this way, the $\mu \mathrm{PL}$ line at $0.9275 \mathrm{eV}(1337 \mathrm{~nm})$ was assigned to the neutral exciton $\left(\mathrm{X}^{0}\right)$ and the line at $0.9252 \mathrm{eV}$ to the biexciton $(\mathrm{XX})$. Positive and negative trions $\left(\mathrm{X}^{+1}\right.$ and $\left.\mathrm{X}^{-1}\right)$ are attributed to optical transitions observed at 0.9290 and 
$0.9231 \mathrm{eV}$, making use of the power dependent evolution [Figs. 3(a) and 3(b)] and the selective optical pumping effect [Fig. 3(c)]. Despite of the fact that single QD emission is demonstrated at very long wavelengths in this sample, typical $\mu \mathrm{PL}$ lines of QDs are broader than in the case of $\mathrm{x}=0.15 \mathrm{LCL}$, as shown in Figs. 2 and 3. Moreover, the emission efficiency of single QDs grown on $\mathrm{In}_{0.30} \mathrm{Ga}_{0.70}$ As LCLs is $20 \%$ of the one from QDs grown on $\operatorname{In}_{0.15} \mathrm{Ga}_{0.85} \mathrm{As}$ LCLs. The lower optical quality of the emission in the $x=0.30$ sample and the larger $\mu \mathrm{PL}$ linewidth in the present case could be explained by the role of the high indium content in the metamorphic layer, leading to an higher plastic relaxation of the strain and an higher density of structural defects ${ }^{10}$ and broadening of the $\mu \mathrm{PL}$ peaks can be related to a spectral diffusion effect by impurities or structural defects. ${ }^{28,29} \mathrm{Nev}$ ertheless there are many methods that can be used to consistently reduce the density of defects close to QDs in metamorphic nanostructures, such as the use of InGaAs graded buffers, ${ }^{30}$ of QD layers acting as defect filters ${ }^{31}$ and of an $\mathrm{ad}$ hoc defect reduction technique. ${ }^{32}$

Therefore, considering that the sample with lower indium content has single QD emission with very good optical quality and efficiencies comparable with those of InAs/GaAs structures, one can conclude that metamorphic InAs QDs are suitable candidates to obtain single QD emission at long wavelengths in GaAs substrates.

In conclusion, we report on the growth and characterization of low density metamorphic QD structures grown on GaAs, showing single QD emission beyond the second telecommunication window $(1.3 \mu \mathrm{m})$ band. The original growth approach is based on the deposition of subcritical InAs coverages on InGaAs metamorphic buffers, that allows to have a low density of $10^{8} \mathrm{~cm}^{-2}$ on the whole sample surface, an advancement with respect to Ref. 12, where the low density was limited to a small area. Experimental evidences sustain the fact that growth of subcritical QDs on InGaAs is rather different from conventional growth on GaAs. Metamorphic QDs allow single photon emission in the telecom windows; moreover, by engineering strain and band discontinuities, as proposed in Ref. 21, these nanostructures may have the potential for tuning emission and other single QD properties.

We gratefully acknowledge the financial support of the Generalitat Valenciana and the Spanish Ministry of Science (Project Nos. PROMETEO/2009/074 and TEC2008-06756C03-03, respectively). One of the authors (D. Rivas) thanks the Ministry of Science for his FPI fellowship.

${ }^{1}$ M. Henini, Handbook of Self Assembled Semiconductor Nanostructures for Novel Devices in Photonics and Electronics (Elsevier, Amsterdam, London, 2008).

${ }^{2}$ D. Bimberg, Semiconductor Nanostructures (Springer, Berlin, 2008).

${ }^{3}$ Z. Yuan, B. Kardynal, R. Stevenson, A. Shields, C. Lobo, K. Cooper, N. Beattie, D. Ritchie, and M. Pepper, Science 295, 102 (2002).

${ }^{4}$ B. Alloing, C. Zinoni, L. Li, A. Fiore, and G. Patriarche, J. Appl. Phys. 101, 024918 (2007)

${ }^{5}$ G. Trevisi, L. Seravalli, P. Frigeri, and S. Franchi, Nanotechnology 20,
415607 (2009).

${ }^{6}$ H. Song, T. Usuki, Y. Nakata, N. Yokoyama, H. Sasakura, and S. Muto, Phys. Rev. B 73, 115327 (2006).

${ }^{7}$ U. Perinetti, N. Akopian, Y. Samsonenko, A. Bouravleuv, G. Cirlin, and V. Zwiller, Appl. Phys. Lett. 94, 163114 (2009).

${ }^{8}$ J. M. Ripalda, D. Alonso-Álvarez, B. Alén, A. G. Taboada, J. M. García, Y. González, and L. González, Appl. Phys. Lett. 91, 012111 (2007).

${ }^{9}$ L. Seravalli, P. Frigeri, G. Trevisi, and S. Franchi, Appl. Phys. Lett. 92, 213104 (2008).

${ }^{10}$ L. Seravalli, P. Frigeri, L. Nasi, G. Trevisi, and C. Bocchi, J. Appl. Phys. 108, 064324 (2010).

${ }^{11}$ Z. Mi and P. Bhattacharya, IEEE J. Sel. Top. Quantum Electron. 14, 1171 (2008).

${ }^{12}$ E. Semenova, R. Hostein, G. Patriarche, O. Mauguin, L. Largeau, I. Robert-Philip, A. Beveratos, and A. Lemaïtre, J. Appl. Phys. 103, 103533 (2008).

${ }^{13}$ P. M. J. Marée, J. C. Barbour, J. F. Van der veen, K. L. Kavanagh, C. W. T. Bulle-Lieuwma, and M. P. A. Viegers, J. Appl. Phys. 62, 4413 (1987).

${ }^{14}$ V. Bellani, C. Bocchi, T. Ciabattoni, S. Franchi, P. Frigeri, P. Galinetto, M. Geddo, F. Germini, G. Guizzetti, L. Nasi, M. Patrini, L. Seravalli, and G. Trevisi, Eur. Phys. J. B 56, 217 (2007).

${ }^{15}$ M. Geddo, G. Guizzetti, M. Patrini, T. Ciabattoni, L. Seravalli, P. Frigeri, and S. Franchi, Appl. Phys. Lett. 87, 263120 (2005).

${ }^{16}$ L. Seravalli, G. Trevisi, P. Frigeri, and C. Bocchi, J. Phys.: Conf. Ser. 245, 012074 (2010).

${ }^{17}$ V. Dubrovskii, G. Cirlin, Y. Musikhin, Y. Samsonenko, A. Tonkikh, N. Polyakov, V. Egorov, A. Tsatsul'nikov, N. Krizhanovskaya, V. Ustinov, and P. Werner, J. Cryst. Growth 267, 47 (2004).

${ }^{18}$ C. Thet, S. Sanorpim, S. Panyakeow, and S. Kanjanachuchai, Semicond. Sci. Technol. 23, 055007 (2008).

${ }^{19}$ N. V. Kryzhanovskaya, A. G. Gladyschev, S. A. Blokhin, Y. G. Musikhin, A. E. Zhukov, M. V. Maksimov, N. D. Zakharov, A. F. Tsatsul'nikov, N. N. Ledentsov, P. Werner, F. Guffarth, and D. Bimberg, Semiconductors 38, 833 (2004).

${ }^{20}$ Y. Jiao, J. Wu, B. Xu, P. Jin, L. Hu, L. Liang, and Z. Wang, Physica E (Amsterdam) 35, 194 (2006).

${ }^{21}$ L. Seravalli, M. Minelli, P. Frigeri, S. Franchi, G. Guizzetti, M. Patrini, T. Ciabattoni, and M. Geddo, J. Appl. Phys. 101, 024313 (2007).

${ }^{22}$ M. Grundmann and D. Bimberg, Phys. Rev. B 55, 9740 (1997).

${ }^{23}$ G. Muñoz-Matutano, B. Alén, J. Martinez-Pastor, L. Seravalli, P. Frigeri, and S. Franchi, Nanotechnology 19, 145711 (2008).

${ }^{24}$ W. H. Chang, H. S. Chang, W. Y. Chen, T. M. Hsu, T. P. Hsieh, J. I. Chyi, and N. T. Yeh, Phys. Rev. B 72, 233302 (2005).

${ }^{25}$ E. S. Moskalenko, K. F. Karlsson, P. O. Holtz, B. Monemar, W. V. Schoenfeld, J. M. Garcia, and P. M. Petroff, Phys. Rev. B 64, 085302 (2001).

${ }^{26}$ J. Gomis-Bresco, G. Muñoz-Matutano, B. Alén, L. Seravalli, P. Frigeri, G. Trevisi, and S. Franchi, New J. Phys. 13, 023022 (2011).

${ }^{27}$ N. I. Cade, H. Gotoh, H. Kamada, and H. Nakano, Phys. Rev. B 73 115322 (2006)

${ }^{28}$ M. Abbarchi, F. Troiani, C. Mastrandrea, G. Goldoni, T. Kuroda, T. Mano, K. Sakoda, N. Koguchi, S. Sanguinetti, A. Vinattieri, and M. Gurioli, Appl. Phys. Lett. 93, 162101 (2008).

${ }^{29}$ P. Alonso-González, B. Alén, D. Fuster, Y. González, L. González, and J. Martínez-Pastor, Appl. Phys. Lett. 91, 163104 (2007).

${ }^{30}$ I. Tångring, H. Q. Ni, B. P. Wu, D. H. Wu, Y. H. Xiong, S. S. Huang, Z. C. Niu, S. M. Wang, Z. H. Lai, and A. Larsson, Appl. Phys. Lett. 91, 221101 (2007).

${ }^{31}$ Z. Mi, J. Yang, P. Bhattacharya, P. Chan, and K. Pipe, J. Vac. Sci. Technol. B 24, 1519 (2006).

${ }^{32}$ L. Y. Karachinskii, T. Kettler, I. I. Novikov, Y. M. Shernyakov, N. Y. Gordeev, M. V. Maksimov, N. V. Kryzhanovskaya, A. E. Zhukov, E. Semenova, A. P. Vasil'Ev, V. M. Ustinov, G. Fiol, M. Kuntz, A. Lochmann, O. Schulz, L. Reissmann, K. Posilovic, A. R. Kovsh, S. S. Mikhrin, V. A. Shchukin, N. N. Ledentsov, and D. Bimberg, Semicond. Sci. Technol. 21, 691 (2006) 